\title{
All good cells come from cells
}

\section{James L. Sherley}

\section{To the Editor:}

A long-standing tenet of biology, omni cellula e cellula (all cells come from cells), seems to have been demolished by research in cloning. It is possible to construct a new viable cell, without mitosis of a pre-existing cell, by combining cell components that are individually acellular. The specific technique, in which isolated somatic-cell nuclei are transplanted into enucleated oocytes to produce cloned embryos, is also called somatic-cell nuclear transfer.

Human cloning has met with resistance because some view it as posing troubling moral issues and foolishly wasting scarce research resources. In a recent commentary, St John and Lovell-Badge offered their perspective on an even more disturbing development in human cloning research (Nature Cell Biology, 9, 988-992, 2007), namely, the proposal to create embryos of mixed species origin, produced with human cell nuclei and enucleated animal oocytes. It is their position that these unnatural cells may be used to produce human-animal embryonic stem cells (h-aESCs).

The authors' stated purpose is to "alleviate public fears and justify why the work is necessary". Their central justification is that human-animal cloned embryos (h-aCLEs) may serve as research models for developing techniques that would make human cloning more efficient for the production of human embryonic stem cells (hESCs). Improving the efficiency of human cloning would address the shortage of human oocytes required for production of humanhuman zygotes.

James L. Sherley is in the Programs in Regenerative Biology and Cancer at the Boston Biomedical Research Institute, 64 Grove Street, Watertown, MA 02472, USA.

e-mail: sherleyj@bbri.org
St John and Lovell-Badge have devoted much of their article to detailing obvious problems that are very likely to contribute to the malfunction of h-aCLEs. However, they do so only from the perspective of problems caused by heterologous mitochondria. They have completely overlooked the certain aberrant and detrimental effects of the reprogramming of transplanted human genomes by animal oocyte factors. The authors' main argument is that the research should be done to study defects in h-aCLEs and the h-aESCs that may be derived from them. Their stated position is, "Of course, before we can determine whether single or cross-species cybrid embryos and ESCs are useful, a considerable amount of research must be performed". In reality, huge volumes of research on oocyte function and basic cellular and molecular biology must be ignored to justify h-aCLE and h-aESC research. Not a single new experiment is necessary to know with certainty that human-animal cloning will not provide faithful models for human-human cloning. This is also a particularly troubling state of affairs, given the increasing scepticism regarding the actual therapeutic potential of human-human cloning.

The authors' treatment of the question whether h-aCLEs will be human is a contrivance that they use to avoid altogether this question of great gravity. They prescribe a definition of 'human' that is based on the molecular content of cells. According to this definition, cells with more human molecules are more human. Thus, they insinuate that h-aCLEs will only be partially human at the time of their destruction to produce haESCs. Of course, h-aCLEs will be human in species because of their human genome. Nothing else is required to define them as human, no matter how aberrant they may be as a result of activation of their genome by animal oocyte factors. St John and LovellBadge are correct when they ask, "If they are not human, what are they?" The only answer to this rhetorical question is a factual one, "Human, of course!"

At the end of their commentary, the authors' intentions become clearer. They ask readers, "Why would anyone choose to prevent any form of research that harms no one and might allow such breakthroughs to take place?" However, the problem is that if research in human-animal cloning succeeds in producing viable h-aCLEs, these human embryos will be harmed to produce cells whose ultimately heterologous and aberrant character will preclude advancing human-human cloning, and will not provide new, effective medical therapies. So perhaps we should reconsider whether cloning has demolished a monumental tenet in biology after all, as really, only a minor refinement of the original tenet has occurred - omni cellula bona e cellula (all good cells come from cells).

We encourage the submission of correspondence on topical issues of relevance to the cell biologist, be it related to policies, ethics or research. See http://www.nature.com/ncb/ authors/article_types/index.html for more information. 